\title{
LEIS DE POTÊNCIA OU ESCALA: SUA APUCAÇÃO AO FENÔMENO ESPORTIVO
}

Juan Manuel García-Manso' ${ }^{1}$ jgarcia@ def.ulpgc.es Juan Manuel Martín-González ${ }^{1}$ jgonzalez@ dfis.ulpgc.es

doi:10.3900/fpj.7.3.195.p

García-Manso JM, Martín-G onzález JM. Leis de potência ou escala: sua aplicação ao fenômeno esportivo. Fit Perf J. 2008 mai-jun;7(3):195202.

\section{RESUMO}

O s sistemas que encontramos na natureza se organizam de forma hierárquica, ou seja, se configuram em estratos que correspondem a escalas características de tempo, longitude ou energia. Por escala nos referimos à dimensão espacial ou temporal de um fenômeno e há três aspectos a considerar: a escala característica, o efeito de escala e 0 escalamento. A lei de escala, que segue muitos fenômenos naturais, é descrita por leis de potência, que são expressões matemáticas do tipo $Y=c X^{b}$; onde $X$ e $Y$ são duas variáveis, quantidades ou observações, $c$ é uma constante e b é 0 expoente de escala. Uma expressão deste tipo tem duas propriedades fundamentais: 1) sua transformação logarítmica se transforma em uma reta $[\log (Y)=\log (c)+b \log (X)] ; 2$ ) é invariante a mudanças de escala. Há, basicamente, quatro âmbitos nos quais estas funções se utilizam: no estudo de sistemas de tipo biológico (leis alométricas); na geometria fractal, onde se usam para a determinação das dimensões fractais; em certos tipos de redes complexas; e no estudo das distribuições de probabilidade com comportamentos livres de escala. Sua utilidade no esporte é recente e abre uma nova linha metodológica na pesquisa aplicada, permitindo abordar, com ferramentas simples, uma infinidade de temas relacionados com a estrutura do esporte, sua manifestação na competição e a forma de treiná-lo.

\section{PALAVRAS-CHAVE}

Corrida, Modelos Lineares, Avaliação.

${ }^{1}$ Universidad de Las Palmas de Gran Canaria - ULPGC - Departamento de Educación Física - Las Palmas de Gran Canaria - España 


\section{LAWS OF Potency OR SCALE: ITS APPLICATION TO THE SPORTIVE PHENOMENON}

\section{ABSTRACT}

The systems that we find in nature are organized in a hierarchical way, which means that they are configured in layers, to the ones that characteristic scales of time, longitude and energy are corresponded. When we refer to "scale" we're talking about the space or time dimension of a phenomenon and there are three aspects to consider: the characteristic scale, the scale effect and the scaling. The scales law, which follows many natural phenomenon, is described by the laws of potency, which are mathematic expressions, such as $Y=c X^{b}$; where $X$ and $Y$ are two observable variables, $c$ is a constant and $b$ the scale exponent. $\mathrm{Na}$ expression like this has two fundamental properties: 1 ) its logarithmic transformation is converted into a straight $\operatorname{line}[\log (Y)=\log (c)+b \log (X)] ; 2)$ it is invariable to scale changes. There are, basically, four ambits in which these functions are used: in the biological type systems (allometric laws), of fractal geometry, where it is used for the determination of fractal dimensions; in certain types of complex nets and in the study of the distributions of probability with free scale behavior. Its utility in sports is recent and opens a new methodological line in the applied research, allowing to approach, with simple tools, an infinity of subjects related to the structure of sports, its manifestation in the competition and the way to train it.

\section{KEYWORDS}

Running, Linear Models, Evaluation.

\section{LeYES DE POTENCIA O ESCALA: SU APLICACIÓN AL FENÓMENO DEPORTIVO}

\section{RESUMEN}

Los sistemas que encontramos en la naturaleza se organizan de forma jerárquica, es decir, se configuran en estratos a los que corresponden escalas características de tiempo, longitud o energía. Por escala nos referimos a la dimensión espacial o temporal de un fenómeno y hay tres aspectos a considerar: la escala característica, el efecto de escala y el escalamiento. La ley de escala que sigue muchos fenómenos naturales, se describe por leyes de potencia, que son expresiones matemáticas del tipo $Y=C X^{b}$; donde $X$ e $Y$ son dos variables, observables, $c$ es una constante y $b$ el exponente de escala. Una expresión de este tipo tiene dos propiedades fundamentales: 1) su transformación logarítmica se convierte en una recta $[\log (Y)=\log (c)+b \log (X)] ; 2)$ es invariante a cambios de escala. Hay básicamente cuatro ámbitos en los que estas funciones se utilizan: en el estudio de sistemas de tipo biológico (leyes alométricas); en la geometría fractal, donde se usan para la determinación de las dimensiones fractales; en ciertos tipos de redes complejas; en el estudio de las distribuciones de probabilidad con comportamientos libres de escala. Su utilidad en el deporte es reciente y abre una nueva línea metodológica en la investigación aplicada, permitiendo abordar, con herramientas sencillas, una infinidad de temas relacionados con la estructura del deporte, su manifestación en la competición y la forma de entrenarlo.

\section{PALABRAS CLAVE}

Carrera, Modelos Lineales, Evaluación.

\section{INTRODUÇÃO}

Por escala nos referimos, geralmente, à dimensão espacial ou temporária de um fenômeno. A gama de magnitudes no universo é imensa, desde tamanhos microscópicos até anos-luz; desde fatos que duram microssegundos até fatos cuja evolução se mede em bilhões de anos; velocidades; energias liberadas; etc. Algo similar ocorre com as diferentes escalas usadas, sendo uma realidade que cada elemento ou nível da estrutura se rege com escalas diferentes, não sempre fixas. Tanto os animais como os sistemas que o compõem e as organizações sociais, não podem se fazer muito maiores ou muito menores sem experimentar mudanças fundamentais na sua estrutura ou conduta. Isto pode ser visto na construção de um edifício, onde seu tamanho, sem dúvida, é afetado, tanto pelos materiais de construção como por tudo o que o rodeia: vizinhos, climatologia, normas municipais, etc. Uma cidade não é um povo grande. 0 s serviços, indústrias, padrões de convivência, bairros, circulação, etc., requerem métodos de administração muito diferentes. Pensemos, por exemplo, nas diferenças entre organizar uma competição esportiva em nível local ou em nível 
internacional; ou treinar para melhorar a condição física de um sedentário, frente ao treinamento de alto rendimento. Algo similar também ocorre com o rendimento e as características morfológicas dos praticantes.

$\mathrm{Na}$ mesma geometria fundamental, à medida que um objeto aumenta de tamanho, seu volume aumenta mais rápido que sua superfície, e as propriedades que dependem do volume, como a capacidade e o peso, mudam de proporção com as propriedades que dependem da área, como a força dos suportes ou a atividade de superfície (pensemos em força muscular e seção transversal ou volume muscular). Sistemas como os pulmões, os vasos sanguíneos ou as raízes das árvores, intercambiam substâncias com seu ambiente através de superfícies altamente ramificadas e cuja geometria tem características fractais.

N as escalas, há três aspectos a considerar: a escala característica; o efeito de escala; e o escalamento. Por escala característica entendemos a escala distintiva ou a categoria de escala de um fenômeno que, de forma alguma, caracteriza seu comportamento. 0 tamanho de uma pessoa, de um animal ou de uma casa, são exemplos. Também podemos considerar a freqüência de um fenômeno, como o ciclo anual das estações, a duração do dia, as revoluções por minuto de um motor o ritmo cardíaco basal de uma pessoa ou os pulsos de um hormônio. As escalas características são intrínsecas aos fenômenos que concernem, mesmo que detectá-las possa ser subjetivo, e podem também ser percebidas como níveis em hierarquia. A escala intrínseca se refere à escala a qual um padrão ou processo opera. Em geral, é melhor considerar a escala observada de um fenômeno como o resultado da interação entre 0 observador e a escala inerente do fenômeno. 0 efeito de escala tem a ver com a mudança no resultado de um estudo, devido a mudanças na escala à qual conduz dito estudo. Assim, não é correto extrapolar resultados obtidos em estudos cujas amostras são sujeitos sedentários ou moderadamente ativos, ao treinamento de esportistas com um nível de rendimento muito elevado. O s efeitos da mudança de escala, portanto, podem ser muito importantes na amostragem de dados ou em desenhos experimentais. Enquanto o escalamento, de forma muito geral, tem a ver com as conseqüências de mudar de tamanho.

No entanto, também se encontram fenômenos livres de escala, ou que carecem de escala característica. Um geólogo, por exemplo, quando fotografa uma rocha ou uma paisagem, usa uma moeda, um lápis ou o martelo para definir a escala, devido ao fato de que um processo geológico permanece, à simples vista, mais ou menos igual, tanto se o olhamos em uma escala grande quanto em uma pequena. Isto é, não há padrões que o olho possa identificar como tendo um tamanho típico. Também há fenômenos que parecem se repetir, ou ser o mesmo, so bre uma ampla categoria de escalas, como uma couve-flor, os alvéolos pulmonares, etc. Tais objetos, às vezes, são chamados autoimilares ou fractais. Assim, paisagens, linhas de costa ou partes do córtex terrestre são invariantes à escala, isto é, parecem os mesmos a quase quaisquer escalas. C uriosamente, porém, também são os processos que Ihes vão dando forma, como a erosão ou a sedimentação. Mais adiante ampliaremos estes temas.

\section{Leis de potência (LP) ou de escala}

A lei ou o comportamento à escala que seguem muitos fenômenos naturais, se descrevem por LP. Estas se manifestam em numerosos fenômenos, freqüentemente fractais, onde uma grande quantidade de elementos interage entre si para produzir uma estrutura em nível superior. Estes sistemas evoluem longe do equilíbrio e, com freqüência, são altamente dissipativos. Eles são descritos mediante expressões matemáticas do tipo:

$$
Y=c X^{b}
$$

$O$ nde $X$ e $Y$ são duas variáveis, quantidades ou observações, c é uma constante (também pode se entender como uma constante de normalização), e b é o expoente de escala. Não devemos confundir esta expressão com a função exponencial que tem a forma:

$$
Y=c a^{x}
$$

Convém levar em conta que uma lei exponencial tende a zero, ou a algum outro valor, de forma asintótica e de maneira muito mais rápida do que uma LP, também se escrevendo como:

$$
Y \sim X^{b}
$$

onde se lê como "proporcional a".

Uma expressão deste tipo tem duas propriedades chaves:

1 a propriedade

Se tomarmos o logaritmo na equação (1), nos fica

$$
\log (Y)=\log (c)+b \log (X)
$$


Figura 1 - Ajuste de uma Power Law a uma série de dados

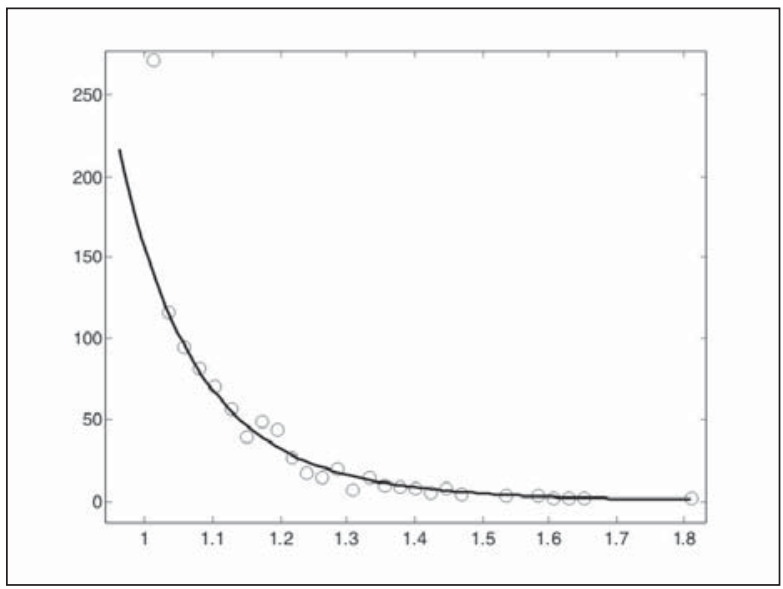

que é a equação de uma reta de inclinação b. Isto é, se, em lugar de representar os valores de $X$ contra os de $Y$ em um gráfico, representamos seus logaritmos $\log (X)$ contra $\log (Y)$, o que vemos é uma linha reta.

Portanto, temos uma forma rápida de rastrear se uma série de dados segue uma LP: representamos seus logaritmos e estimamos até que ponto o resultado pode ser considerado ou ajustado por uma linha reta. 0 utra vantagem é a quantidade de resultados estatísticos (por exemplo, análise de regressão) que se desenvolveram para ajustar e modelar comportamentos lineares. Por isso, em quase todos os trabalhos e artigos vê-se resultados em gráficos logarítmicos, com os correspondentes parâmetros estatísticos que caracterizam o ajuste (desvio quadrático médio, $R^{2}$, etc.).

Na Figura 1 vê-se o ajuste de uma LP a uma série de dados. A figura seguinte é um gráfico "duplamente logarítmico" (o logaritmo de uma magnitude frente ao logaritmo da outra) dos dados anteriores. As duas representações são equivalentes.

2a propriedade

A LP é invariante a mudanças de escala. Suponhamos que, na expressão da LP (1) mudamos a escala (reescalamos) da variável multiplicando-a por um fator z, como sucede ao mudar a escala de um mapa, ou ao passar de metros a quilômetros, etc. $A$ variável $X$ se transforma em zX, e o novo valor da variável Y será:

$$
Y^{\prime}=c(Z X)^{b}
$$

Q ue é o mesmo, depois que um pouco de álgebra, que

$$
Y^{\prime}=c\left(Z^{b}\right) X^{b}=\left(c Z^{b}\right) Y
$$

Figura 2 - Representação duplamente logarítmica (loglog) da mesma série da Figura 1

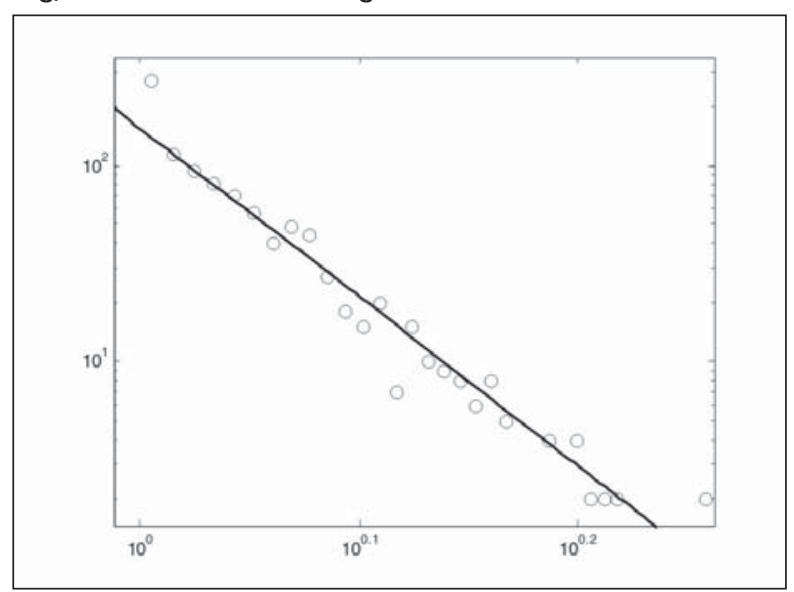

Como vemos, o resultado é uma função do mesmo tipo, só que a constante é agora $c z^{b}$. N este caso, se costuma dizer que qualquer mudança de escala é "absorvida" na constante de normalização, e a forma da função permanece invariante. Esta propriedade é conhecida como invariância em escala (scaling invariance) e os fenômenos com este tipo de comportamento são denominados livres de escala (scale free). Uma série de dados que se distribuam desta maneira, ou que se expressem seguindo uma lei deste tipo, não têm longitudes (magnitudes) características. Savaglio $\&$ Carbone ${ }^{1}$ utilizam este princípio para analisar o ponto de corte nas provas atléticas de corrida ou a forma com que se comportam os rendimentos nestas especialidades do atletismo.

Há, basicamente, quatro âmbitos nos quais estas funções se utilizam: no estudo das leis de escala em sistemas de tipo biológico, onde se denominam leis alométricas; dentro da geometria fractal, usada para a determinação das dimensões fractais; ao analisar certos tipos de redes complexas; e no estudo das distribuições de probabilidade com comportamentos livres de escala.

\section{Exemplos de aplicação de LP nos campos da atividade física e do esporte}

No estudo do rendimento esportivo, as LP não foram muito utilizadas no passado. Mas, como assinalam Katz \& $K a t^{2}$, os recordes mundiais são medidas bem estabelecidas e muito padronizadas, e as LP podem ser muito úteis para investigar o rendimento humano. Por exemplo, nas modalidades de corridas atléticas, a distância perco rrida d e o tempo de corrida t seguem uma lei de escala

$$
t(d)=c d^{b}
$$


onde $b$ é 0 expoente escala ${ }^{2}$, que parece ser 0 mesmo desde a corrida de $100 \mathrm{~m}$ até a de $10.000 \mathrm{~m}$ ou a maratona.

Assim, o expoente b medido em 1925 deu um valor de 1,141 e em 1995 foi de 1,123. Um resultado parecido se encontrou para nadadores, tanto para homens como para mulheres. Isto significa que existe invariância em escala para o rendimento humano, isto é, se mudamos a distância d a outro valor cd, a equação fica

$$
t(c d)=c^{b} t(d)
$$

ou, o que é o mesmo, o quociente entre os tempos para duas distâncias diferentes

$$
t(c d) / t(d)=c^{b}
$$

Figura 3 - Logaritmo do tempo vs. a distância nas meIhores marcas das corridas entre os $200 \mathrm{~m}$ e a maratona. Inclui-se o ponto de corte e o valor das ladeiras de ambas retas.

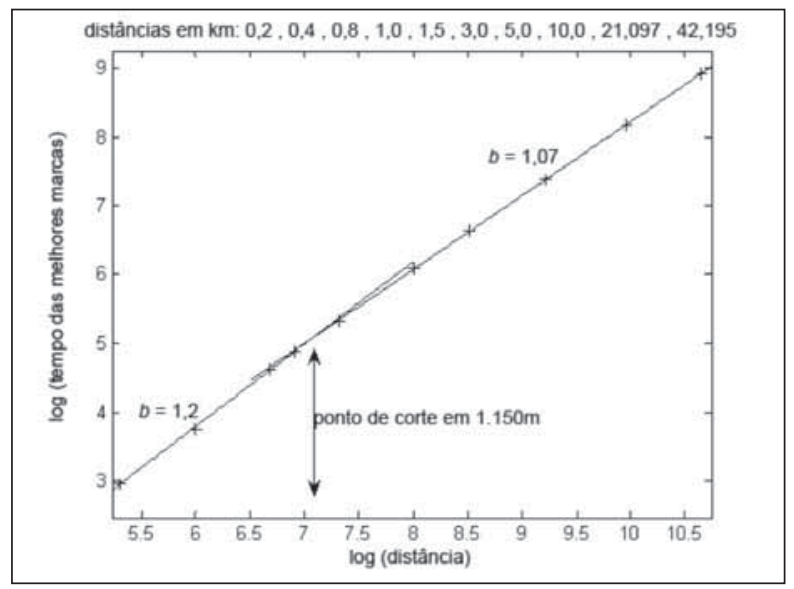

Figura 5 - Representação gráfica da velocidade vs. posto ocupado pelo corredor no ranking de $1500 \mathrm{~m}$. Curva realizada em forma invertida à da Figura 4.

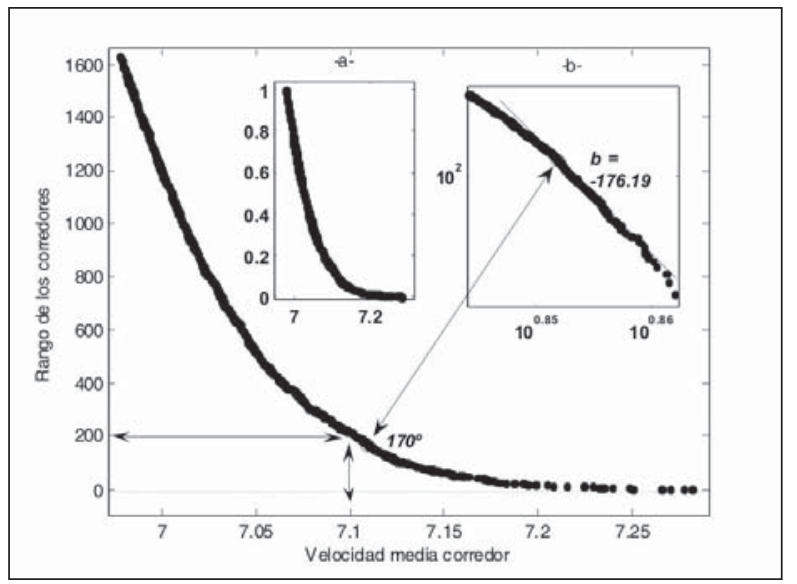

depende só do índice de escala b.

Como a invariância de escala implica invariância das propriedades estatísticas do sistema sobre todas as escalas, não é possível definir uma escala característica. Este comportamento é típico no ramo da Física que estuda os sistemas estatísticos em pontos críticos, como as transições de fase. Como assinalam Savaglio \& Carbone ${ }^{\mathbf{1}}$, o comportamento dos esportistas durante as corridas atléticas ou as provas de natação pode ser considerado um fenômeno crítico. Para entendê-lo, nos fixemos na Figura 3. No eixo x são representados os logaritmos dos valo res das distâncias percorridas em cada prova, desde os $200 \mathrm{~m}$ até a maratona. No eixo y estão os logaritmos dos melhores tempos obtidos em competições oficiais.

Figura 4 - Velocidade vs. faixa que ocupa um corredor no ranking das 1633 melhores marcas all-time da distância de $1500 \mathrm{~m}$. No quadro se mostra o gráfico duplamente logarítmico dos valores da mesma série de dados. Nota-se o ponto de corte no posto 170 do ranking.

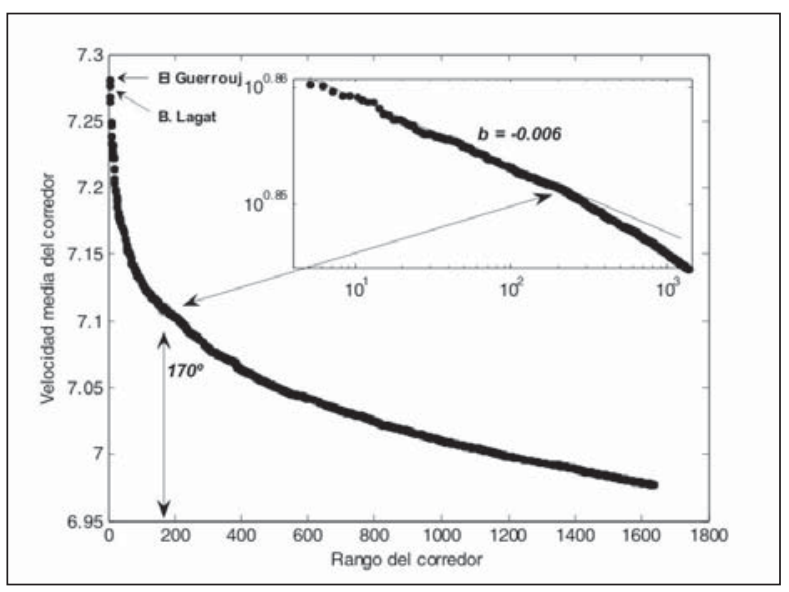

Figura 6 - Diagrama de barras do ranking mundial de $1500 \mathrm{~m}$ para um número de intervalos $\mathrm{n}=30$.

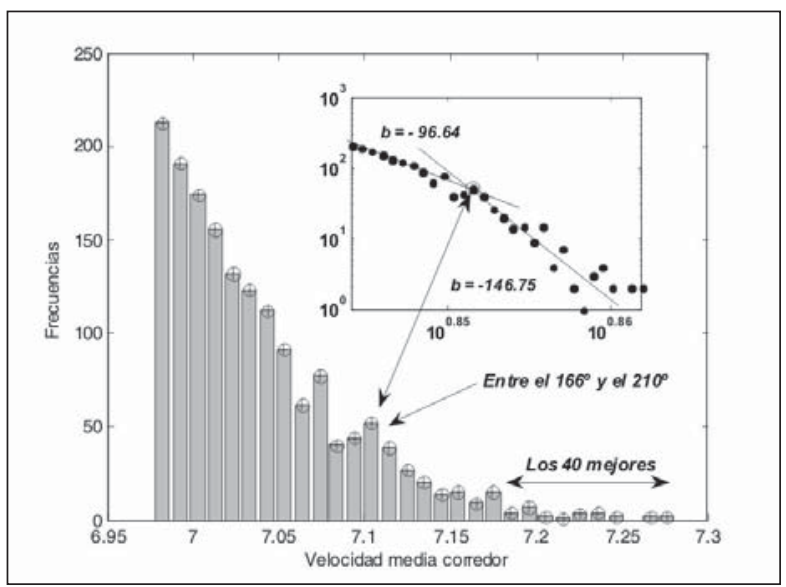


Figura 7 - Histograma mostrando a distribuição de todos os jogadores do mundo com um ELO superior a 2200. No quadro se mostram os logaritmos dos parâmetros representados no histograma (eixo x: valor do ELO; eixo y: freqüência).

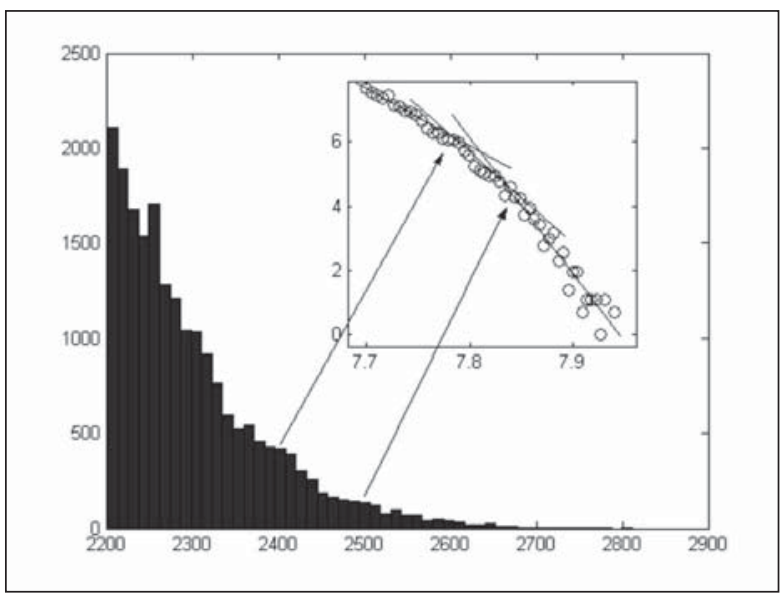

Mesmo que os dados, à primeira vista, pareçam seguir, em conjunto, uma linha reta, na curva que vemos no gráfico se observa uma mudança, um "joelho" ou "cotovelo", perto dos $1.000 \mathrm{~m}$, que define o ponto de corte de duas retas com expoentes de escala diferentes. Há de se ter presente que os dados de distância representados nos eixos do gráfico são logarítmicos. A transição entre os dois expoentes de escala, como observam Savaglio \& Carbone ${ }^{1}$, define um ponto perto dos 1.150 m (Figura 3), que corresponde, para os autores, à mudança entre corridas de curta distância, onde predomina o metabolismo anaeróbico (força), e as corridas de resistência, com o comportamento aeróbico característico necessário para correr longas distâncias.

Às vezes, é melhor o uso da velocidade média de corridas, isto é, o tempo empregado dividido pela distância, já que esta magnitude está mais relacionada com a energia consumida, ou a potência, através do quadrado da velocidade. A lei de escala é

$$
v=c^{\prime} d^{b^{\prime}}
$$

$$
\text { sendo } b^{\prime}=1-b^{-1} \text {. }
$$

Com o objetivo de ilustrar o uso das LP e como trabalhar com elas, nos centraremos em um exemplo obtido do esporte: a corrida de $1.500 \mathrm{~m}$ (dados próprios não-publicados). A escolha se deve a várias razões: esta é uma das provas atléticas mais populares; está bem documentada; e há muito bons corredores, inclusive espanhóis. Além disso, não é uma corrida de resistência
Figura 8 - Seqüência dos jogadores de xadrez $(n=800)$ com um ELO superior a 2500. No quadro os logaritmos destes valores. $\mathrm{O}$ ponto de corte indica onde marca a diferença entre os bons jogadores e os que podem ser considerados como super-elites.

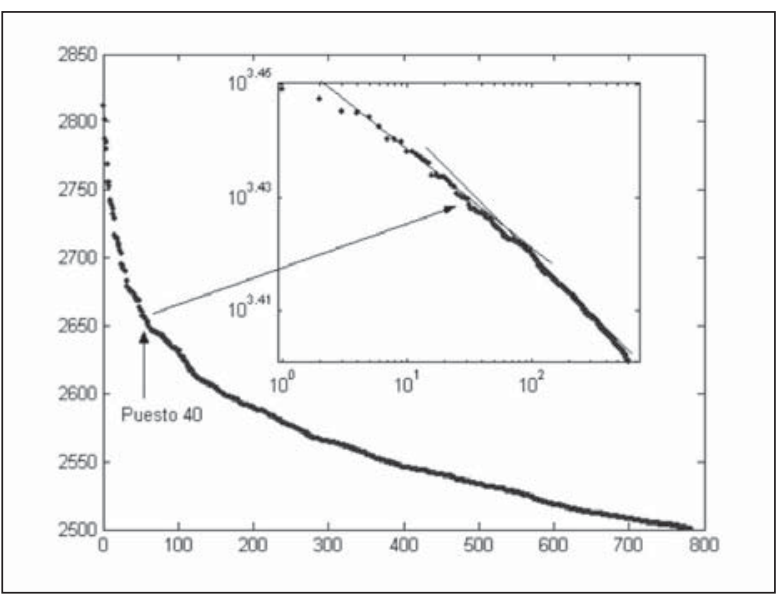

pura, mesmo que se encontre além do valor assinalado antes, $1.150 \mathrm{~m}$, que separe ambas as provas.

Aqui, analisaremos só alguns aspectos competitivos, nos baseando no estudo das melhores marcas obtidas pelos melhores corredores em todos os tempos. Para isso, buscamos os melhores resultados de 1.633 corredores que têm obtido marcas a cima de certo valor, transformados à velocidade, isto é, o melhor tempo em segundos dividido por $1.500 \mathrm{~m}$.

Estes valores, em $m \cdot s^{-1}$, foram colocados em ordem decrescente. Assim, o de posto 1 tem a melhor marca (neste caso, corresponde a El Guerrouj), com posto 2 a segunda melhor marca (em poder, até esse momento, de B. Lagat), e assim sucessivamente.

$\mathrm{Na}$ Figura 4 se mostram os resultados. No quadro damos o correspondente gráfico duplamente logarítmico, aparecendo o padrão em linha reta típico das LP, assim como o valor do expoente $b$.

O bserva-se como o gráfico 4 mostra um ponto de corte no posto 170 , assinalado com setas, que separa claramente dois comportamentos. Isto é, parece que o sistema vem determinado, pelo menos, por duas leis de escala. Uma reflete o comportamento dos primeiros 170 corredores, ou seja, a "elite", e outra para o resto de corredores de nível internacional.

Aplicando uma LP aos 170 primeiros corredores

$$
v(i)=c_{1} i^{-b}
$$

sendo i a categoria i $=1,2, \ldots, 170 ; \mathrm{v}(\mathrm{i})$ a velocidade média do corredor que ocupa o posto número i 
no ranking; e $c_{1}$ é uma constante de normalização em m. s. $^{-1}$.

Vejamos de outra maneira. $\mathrm{Na}$ Figura 5 se mostram os valores da velocidade no eixo xe os postos dos corredores no eixo $y$, isto é, invertemos o gráfico anterior.

Se nos fixamos no gráfico, duas setas assinalam um valor concreto: o posto 200, ao qual corresponde um valor da velocidade de $7,1 \mathrm{~m} \cdot \mathrm{s}^{-1}$. Este tipo de curva se pode interpretar da seguinte maneira: acima do valor da velocidade $7,1 \mathrm{~m} \cdot \mathrm{s}^{-1}$ há 200 corredores de um total de 1.633; ou que $12,24 \%$ dos corredores superam a velocidade de $7,1 \mathrm{~m} \cdot \mathrm{s}^{-1}$. Também o podemos ver como probabilidades. Para isso, dividimos to das as categorias pelo total, ficando como se vê no quadro (a) do gráfico. Como $200 / 1663=0,122$, poderemos dizer que a probabilidade de um corredor obter um valor superior a $7,1 \mathrm{~m} \cdot \mathrm{s}^{-1}$ é de 0,122 . Desta maneira, a curva do gráfico se interpreta como uma distribuição de probabilidade acumulada, ou seja:

$$
\mathrm{P}(>\mathrm{vc})=\mathrm{C}_{2} \mathrm{v}^{-b}
$$

que podemos ler como "a probabilidade de um corredor alcançar um valor acima de um fixo $v_{c}$ ou de ocupar um posto acima de um valor determinado (como o 200), decai inversamente como uma potência da velocidade".

U ma terceira forma de vê-lo é através do histograma ou da distribuição de probabilidades. Para isso, dividimos a diferença entre o máximo e mínimo valor da velocidade em um número de intervalos $\mathrm{n}$. A seguir, contamos quantos corredores têm marcas em cada intervalo, isto é, as freqüências de cada intervalo. A Figura 6 , através de um diagrama de barras, mostra essas freqüências para um número de intervalo $n=30$.

É notável a aparição de intervalos mais povoados que seus vizinhos, dos quais destacamos dois: um em torno do valor 7,1 , que corresponde ao postos entre 0166 e 210 , e outro que representa os postos em torno de 40 . García-Manso et al. ${ }^{3}$ assinalam que estes valores correspondem às barreiras, isto é, àqueles valores que um esportista tende a alcançar à medida que melhora seu rendimento. Em outras palavras, existem metas fixas ou marcas determinadas que funcionam, de alguma maneira, como atrativos e ao mesmo tempo como filtros. De fato, quando um esportista bate um recorde importante está abrindo o caminho dos demais para uma nova barreira. No quadro, representam-se os logaritmos das velocida- des frente às freqüências, aparecendo o conhecido joelho, e os valores dos expoentes de escala obtidos para as duas retas.

0 esporte é, portanto, um âmbito onde podemos encontrar este tipo de distribuição. Há alguns trabalhos em esportes individuais, como é o caso do atletismo ${ }^{2,3,4,5,6}$. Em esportes de equipe também parece que se dão as LP. Malacarne $\&$ Mendes ${ }^{7}$ consideram os maiores artilheiros de campeonatos de liga de diferentes países onde o futebol é um fenômeno das massas. Assim, contam o número de jogadores que marcaram $\mathrm{n}$ gols, depois os que marcaram $n-1$, etc., e encontra uma LP. A distribuição de gols por jogador segue, segundo estes autores, um processo de "difusão anômala" ou LP ZipfMandelbrot. Também Greenhough et al. ${ }^{8}$ destacam a relação existente entre a distribuição dos gols que se conseguem em futebol com estatísticas de fenômenos extremos (filas longas).

Neste tipo de competições também se encontraram resultados parecidos ao analisar o tempo médio que dura um treinador em uma equipe de certa categoria, em vários esportes. Achamos interessante comentar 0 artigo de Aidt et al. ${ }^{9}$, no qual mostram como o tempo que um treinador permanece no comando de equipes de futebol, beisebol e futebol americano de muitos países, segue uma LP com valores dos expoentes entre 2 e 3 . Para isso, trabaIharam sobre um banco de dados com um total de 7.183 treinadores onde, em alguns casos, os dados têm 130 anos de idade, centrando-se no tempo, em anos, que cada treinador permaneceu em uma equipe. Analisam diferentes períodos: 1874-1900, 1874-1920, 1874-1966, 1874-1980 e 1874-2005. Encontraram que, à medida que o sistema evolui, a distribuição vai se ajustando cada vez melhor a uma LP. Por isso, deduzem que as LP, neste contexto, são um fenômeno emergente, ou seja, emergem como conseqüência da evolução do sistema; um resultado já obtido para outros sistemas complexos. De todas as atitudes, convém assinalar que os autores não consideram aqueles treinadores despedidos no seu primeiro ano ou temporada, pois não parece haver muitas razões para isto proceder, salvo por alguma casualidade. Por outra parte, no seu primeiro ano de trabalho costuma haver uma espécie de "lua-de-mel", dentro da qual os treinadores formam uma equipe e, na opinião dos autores, não costumam ser julgados até a segunda temporada. 
Por países, salvo na França, os dados seguem uma distribuição LP. Vejamos os resultados obtidos para os expoentes de escala, no artigo citado. No Futebol (desde 1874 até 2005, mesmo que não em todos os países; por exemplo, na Espanha desde 1910): Inglaterra, -2,23; Suíça, -2,93; França ---; Espanha, -2,75; e Alemanha, -2,51. Em beisebol: USA, -2,63; e Japão, $-2,41$. Em Futebol americano, $-2,08$. 0 s auto res pensam que 0 rendimento dos treinadores se mede pelo das equipes que lideram; trabalham em um ambiente muito competitivo e são, geralmente, despedidos quando seu crédito cai sob certo valor umbral ou, pelo contrário, são comprados por outras equipes se seu sucesso é suficientemente alto. Uma pergunta fica no ar: o que acontece no futebol francês?

0 xadrez também responde a este tipo de comportamento. Em um recente artigo, Blasius \& Toenjes ${ }^{10}$ encontram que as aberturas de xadrez mais utilizadas, tanto por jogadores torcedores como por grandes professores, se distribuem de acordo com uma LP que tem um expoente que aumenta à medida que 0 jogo avança. Após ler este trabalho, nós nos perguntamos se os rankings neste esporte também seguem uma LP. Lembremos que o nível de rendimento do jogador de xadrez se mede por um procedimento que atribui um valor denominado ELO a cada esportista. Para responder a esta questão, selecionamos uma lista de 20.600 jogadores com um valor de ELO superior a 2200. O ELO é um sistema matemático, elaborado pelo Prof. Arpad Elo (Professor de Física da Universidade de Milwaukee), para a avaliação do rendimento dos jogadores de xadrez. Com ele se pode saber, sem conhecer um jogador, qual é seu nível de jogo e permite realizar classificações dos jogadores. 0 ELO é avaliado em cada competição e leva em conta 0 ELO prévio de cada rival para aumentar ou diminuir o próprio, segundo o resultado em função do rival e 0 resultado da partida. Para obter um valor alto de ELO é necessário competir com jogadores que possuem ELO alto. Isto implica, portanto, que se há de competir em alto nível, em torneios internacionais e com muitas interações entre jogadores de grande nível, o qual limita o número de jogadores de elite.

$\mathrm{Na}$ Figura 7, observamos como o ELO parece seguir uma LP. Para comprová-lo, representamos no quadro os logaritmos dos parâmetros do histograma (eixo $\mathrm{x}$ : valor do ELO ; eixo y: freqüência). O bservemos como, aparentemente, há três linhas (tendências) diferenciadas que apresentam pontos de corte em valores de ELO entre 2400 e 2500 . Estes cortes respondem a níveis de competição diferentes. Assim, acima dos 2400 há uma elite de jogadores que inclui, aproximadamente, os 800 melhores jogadores mundiais. Se nos centrarmos apenas nos de valor superior a 2500 (Figura 8) e os representarmos por ordem de categoria (o primeiro é o de valor mais alto, o segundo o seguinte, etc.), obteremos o gráfico anterior (Figura 7). No quadro se mostra a representação duplamente logarítmica, que também segue um duplo comportamento linear, com uma "super-elite" que abrange os 40 primeiros, com valores de ELO acima de 2650 , onde se deve dar a máxima rivalidade.

\section{DISCUSSÃO}

À luz dos exemplos mostrados, demonstra-se que também é possível abordar o fenômeno esportivo a partir de uma perspectiva diferente da qual propõem os modelos tradicionais (óptica reducionista). Afrontar o esporte desde uma óptica diferente, permite enriquecer nosso corpo de conhecimento e, ao mesmo tempo, aumentar o pool de instrumentos de medição, avaliação e caracterização que nos levam a compreender o processo pela óptica dos sistemas complexos não-lineares.

Isto é o que tratamos de demonstrar com a a plicação das leis de escala ou potência ao estudo do fenômeno esportivo em um de seus aspectos (senão o mais importante): o rendimento. Sem dúvida, não é a única metodologia útil, nem sequer a mais potente, mas à luz dos dados se comprova como um instrumento útil e confiável para abordar o fenômeno, contribuindo com aspectos diferentes dos oferecidos pelo modelo biofísico.

\section{REFERÊNCIAS}

1. Savaglio S, Carbone V. Scaling in athletic worlds records. Nature. 2000;404:244.

2. Katz JS, Katz L. Power laws and athletic performance. J Sports Sci. 1999;17:467-76.

3. García-Manso JM, Martín-González JM, Arriaza E, González L. Middle and long distance races viewed from the complexity: macroscopic analysis based on behaviour as a power law. New Studies in Athletics. IAAF. 2006;21(1):17-25.

4. García-Manso JM, Martín-González JM, Dávila N, Arriaza E. Middle and long distance athletics races viewed from the perspective of complexity. J Theor Biol. 2005;233(2):191-8.

5. Alvarez-Ramirez J, Rodríguez E. Scaling properties of marathon races. Physica A. 2006;365(2):509-20.

6. García-Manso JM, Martín-González JM, Da Silva-Grigoletto ME, Vaamonde $D$, Benito P, Calderón J. Male powerlifting performance described from the viewpoint of complex systems. J Theor Biol. 2008;251(3):498-508.

7. Malacarne LC, Mendes RS. Regularities in football goal distributions. Physica A. 2000;286:391-5.

8. Greenhough J, Birch PC, Chapman SC, Rowlands G. Football goal distributions and extremal statistics. Physica A. 2002 dez;316(1-4):615-24.

9. Aidt $T$, Leong $B$, Sgroi $D$, Saslaw W. A power-law distribution for tenure lengths of Sport managers. Physica A. 2006;370(2):697-703.

10. Blasius B, Toenje R. Zipf law in the popularity distribution of chess openings. [submetido em 2007 abr 20]. ArXiv.

Recebido: 08/01/2008 - Aceito: 25/02/2008 